\title{
Orbital hyperfine interaction and qubit dephasing in carbon nanotube quantum dots
}

\author{
Gábor Csiszár ${ }^{1}$ and András Pályi ${ }^{1,2,3, *}$ \\ ${ }^{1}$ Institute of Physics, Eötvös University, H-1117 Budapest, Hungary \\ ${ }^{2}$ MTA-BME Condensed Matter Research Group, Budapest University of Technology and Economics, H-1111 Budapest, Hungary \\ ${ }^{3}$ Kavli Institute for Theoretical Physics China, Beijing, P.R. China
}

(Received 9 September 2014; revised manuscript received 20 November 2014; published 5 December 2014)

\begin{abstract}
Hyperfine interaction (HF) is of key importance for the functionality of solid-state quantum information processing, as it affects qubit coherence and enables nuclear-spin quantum memories. In this work, we complete the theory of the basic HF mechanisms (Fermi contact, dipolar, orbital) in carbon nanotube quantum dots by providing a theoretical description of the orbital HF. We find that orbital HF induces an interaction between the nuclear spins of the nanotube lattice and the valley degree of freedom of the electrons confined in the quantum dot. We show that the resulting nuclear-spin-electron-valley interaction (i) is approximately of Ising type; (ii) is essentially local, in the sense that a radius- and dot-length-independent atomic interaction strength can be defined; and (iii) has an atomic interaction strength that is comparable to the combined strength of the Fermi contact and dipolar interactions. We argue that orbital HF provides a new decoherence mechanism for single-electron valley qubits and spin-valley qubits in a range of multivalley materials. We explicitly evaluate the corresponding inhomogeneous dephasing time $T_{2}^{*}$ for a nanotube-based valley qubit.
\end{abstract}

DOI: 10.1103/PhysRevB.90.245413

PACS number(s): 73.63.Kv, 73.63.Fg, 71.70.Ej, 76.20.+q

\section{INTRODUCTION}

Carbon nanotubes (CNTs) provide a promising platform $[1,2]$ for quantum information processing [3-6]: In nonmetallic CNTs, one or a few electrons can be captured in an electrically defined quantum dot (QD), potentially allowing for coherent control of the electrons' internal (spin and valley) degrees of freedom.

Hyperfine interaction (HF) between the nuclear spins of the lattice and the electrons in the QD can be either a nuisance or an asset in this context. On the one hand, a randomized nuclear-spin ensemble induces decoherence of a spin-based electronic qubit [7-9]. On the other hand, HF is the mechanism that allows for information transfer between the electronic state and the nuclear spins, a critical step for utilizing nuclear spins as long-lived quantum memories [10-14]. Remarkably, the abundance of nuclear spins in the CNT lattice can be increased (decreased) by isotopic enrichment (purification) [15-17] of the spin-half ${ }^{13} \mathrm{C}$ nuclei, which have a natural abundance of $\sim 1 \%$. The fundamental importance of HF in these nanostructures is also highlighted by the possibility of HF-mediated nuclear magnetism in one-dimensional solids [18-20] including ${ }^{13} \mathrm{C}$-enriched CNTs.

Partly motivated by these attractive features, a series of experiments was carried out with clean CNTs, aiming to control and measure the spin and valley degrees of freedom of electrons confined in QDs [16,17,21,22]. Surprisingly, two of these experiments using ${ }^{13} \mathrm{C}$-enriched samples revealed effects compatible with an atomic HF strength that is two orders of magnitude larger than theoretically calculated $[9,23]$ and measured via nuclear magnetic resonance $[24,25]$. The resolution of this discrepancy is an open problem [2], bearing strong relevance for HF-related phenomena in CNTs.

The interesting prospects in quantum information processing and nuclear magnetism, as well as the theory-experiment

\footnotetext{
*andraspalyi@ caesar.elte.hu
}

mismatch of the coupling strength, stimulated efforts [25-30] toward a more complete understanding of HF in carbon-based nanostructures. These works explore the consequences of two of the three basic mechanisms of HF [31], Fermi contact (a.k.a. isotropic) and dipolar, and exclude the third one, orbital HF (OHF) [32]. It should be noted that the consequences of OHF in nuclear magnetic resonance of CNTs [33,34] and graphene [35] have been analyzed.

In this work, we complete the theoretical description of hyperfine effects in CNT QDs by elucidating the role of the OHF. We show that this mechanism provides an effective interaction between a nuclear spin and the valley degree of freedom of the electron: the simple argument (see Fig. 1) is that the binary valley quantum number $K$ and $K^{\prime}$ labels electronic states circulating along the CNT circumference in the clockwise and counter-clockwise directions [36,37], respectively, and therefore the electron has a valley-dependent orbital magnetic moment that feels the dipole magnetic field created by the nuclear spin. Using the envelope-function model (Dirac equation) for the electrons, and focusing on the case where the longitudinal electronic wavelength $\lambda$ exceeds the nanotube radius $R$, we show that the resulting nuclear-spinelectron-valley interaction (i) is approximately of Ising type, (ii) is essentially local, in the sense that a radius- and dotlength-independent atomic interaction strength can be defined, and (iii) has an atomic interaction strength that is comparable to the combined strength of the Fermi contact and dipolar interactions. We argue that the inhomogeneous dephasing time $T_{2}^{*}$ of single-electron valley qubits and spin-valley qubits is affected by the OHF and explicitly evaluate $T_{2}^{*}$ for a valley qubit.

\section{ORBITAL HYPERFINE INTERACTION WITH THE ELECTRONIC VALLEY DEGREE OF FREEDOM}

Here, we provide an analytical description of the OHFmediated coupling between the nuclear spin of a single

${ }^{13} \mathrm{C}$ atom residing in a CNT QD and the valley degree of 


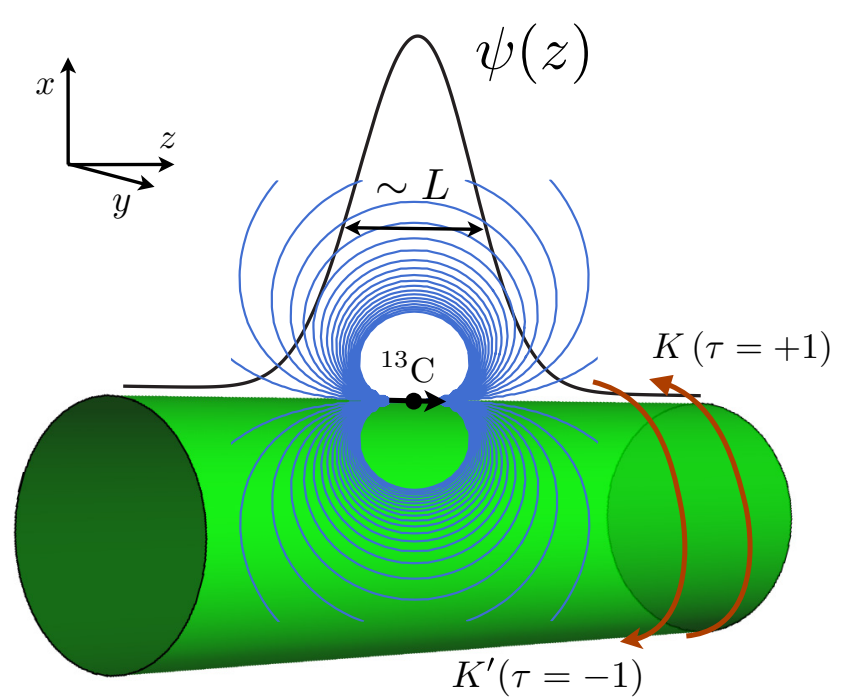

FIG. 1. (Color online) Carbon nanotube quantum dot (QD) with a single spin-carrying ${ }^{13} \mathrm{C}$ nucleus. Arrows labeled $K(\tau=+1)$ and $K^{\prime}(\tau=-1)$ represent the two electronic valley states, moving to opposite directions around the nanotube circumference. The solid black line and $\psi(z)$ represent the longitudinal envelope function characterizing the ground state of an electron confined in the QD. The thin (blue) lines touching the ${ }^{13} \mathrm{C}$ nucleus represent the dipole magnetic field created by the spin of the ${ }^{13} \mathrm{C}$ nucleus.

freedom of a single electron confined to the same QD. To this end, the electron will be described by the canonical envelope-function model [2] of CNTs (Dirac equation). In the terminology introduced by Yafet [32], our approach describes the "long-range" part of the OHF; the "short-range" part of the OHF is shown to be absent in CNTs in the tight-binding framework of Ref. [9]. Our description remains qualitatively valid for any type of spin-carrying nucleus.

The setup and the reference frame are shown in Fig. 1. The spin-carrying ${ }^{13} \mathrm{C}$ nucleus is located at $\boldsymbol{r}_{0}=\left(R, 0, z_{0}\right)$. The nuclear spin has a dipole moment $g_{N} \mu_{N}$, and therefore it creates a vector potential,

$$
\boldsymbol{A}(\boldsymbol{r})=\frac{\mu_{0}}{4 \pi} g_{N} \mu_{N} \frac{\boldsymbol{I} \times\left(\boldsymbol{r}-\boldsymbol{r}_{0}\right)}{\left|\boldsymbol{r}-\boldsymbol{r}_{0}\right|^{3}} \equiv \boldsymbol{I} \times \boldsymbol{v}\left(\boldsymbol{r}-\boldsymbol{r}_{0}\right) .
$$

Here, $g_{N} \approx 1.41$ is the $g$ factor of the ${ }^{13} \mathrm{C}$ nucleus [9], and $\mu_{N} \approx 5.05 \times 10^{-27} \mathrm{~J} / \mathrm{T}$ is the nuclear magneton. The nuclear spin vector operator $\boldsymbol{I}$ is represented by $1 / 2$ times the vector of Pauli matrices.

Technically, OHF between the nuclear spin and the electron arises because the vector potential $\boldsymbol{A}$ created by the nuclear spin enters the kinetic term of the envelope-function Hamiltonian via $\boldsymbol{p} \mapsto \boldsymbol{p}+\boldsymbol{e A}$. The kinetic term, describing an electron in the conduction or valence band, reads [2] $H_{0}+H_{\mathrm{OHF}}$, with

$$
\begin{aligned}
H_{0} & =v_{\mathrm{F}}\left(\tau_{3} \sigma_{1} p_{c}+\sigma_{2} p_{t}\right), \\
H_{\mathrm{OHF}} & =e v_{\mathrm{F}}\left[\tau_{3} \sigma_{1} A_{c}(\boldsymbol{r})+\sigma_{2} A_{t}(\boldsymbol{r})\right] .
\end{aligned}
$$

Here, $c(t)$ is the circumferential (longitudinal) coordinate on the surface of the CNT, and $\sigma_{1,2}$ are sublattice Pauli matrices. Note that our choice of the reference frame (Fig. 1) allows us to use $t$ and $z$ interchangeably. In Eq. (2a), $p_{c}$ is the circumferential momentum quantum number set by the periodic boundary condition along the CNT circumference, whereas $p_{t}$ is the longitudinal momentum operator. In Eq. (2b), we introduced the circumferential and longitudinal projections of the vector potential, $A_{c}(\boldsymbol{r})=\hat{\boldsymbol{c}}(c) \cdot \boldsymbol{A}(\boldsymbol{r})$ and $A_{t}(\boldsymbol{r})=$ $\hat{\boldsymbol{t}} \cdot \boldsymbol{A}(\boldsymbol{r})$, respectively, where $\hat{\boldsymbol{c}}(c)=\left(-\sin \frac{c}{R}, \cos \frac{c}{R}, 0\right), \hat{\boldsymbol{t}}=$ $(0,0,1)$, and $\boldsymbol{r} \equiv \boldsymbol{r}(c, t)=\left(R \cos \frac{c}{R}, R \sin \frac{c}{R}, t\right)$. Form (2) of the Hamiltonian is valid for any chirality; here we focus on CNTs with a finite gap (i.e., $p_{c} \neq 0$ ) allowing for electrostatic QD confinement.

Using Eqs. (1) and (2), the OHF Hamiltonian can be written as

$$
H_{\mathrm{OHF}}=e v_{\mathrm{F}} \tau_{3} \sigma_{1} \varepsilon_{\alpha \beta \gamma} I_{\alpha} v_{\beta}\left(\boldsymbol{r}-\boldsymbol{r}_{0}\right) \hat{c}_{\gamma},
$$

where $\varepsilon_{\alpha \beta \gamma}$ is the Levi-Civita symbol, the Einstein summation convention is used, and the valley-independent term have been omitted, as it is irrelevant for valley dynamics.

For simplicity, we assume $p_{c}>0$ and anticipate that a sign change in $p_{c}$ implies a sign change of the coupling constants $C_{\alpha}$ (defined below). Then an electronic low-energy energy eigenstate in the valley $\tau \in\left(K, K^{\prime}\right) \equiv(+1,-1)$ of the conduction band of the electrostatically defined QD is approximately described by the four-component spinor envelope function

$$
\Psi_{\tau}(c, t)=|\tau\rangle \otimes|\chi\rangle \otimes \frac{e^{i \tau\left(p_{c} / \hbar\right) c}}{\sqrt{2 \pi R}} \psi(t),
$$

where $|\tau=+1\rangle=(1,0)^{T}$ or $|\tau=-1\rangle=(0,1)^{T}$ represents the valley state, $|\chi\rangle=(1,1)^{T} / \sqrt{2}$ characterizes the sublattice amplitudes at the bottom of the conduction band, and $\psi(t)$ is the longitudinal envelope function of the electron. The normalization condition $\int_{-\infty}^{\infty} d t \int_{0}^{2 \pi R} d c \Psi^{\dagger}(c, t) \Psi(c, t)=$ 1 demands that $\int_{-\infty}^{\infty} d t|\psi(t)|^{2}=1$. Note that by writing the envelope function $\Psi_{\tau}(c, t)$ as a product of a circumferential and longitudinal component in Eq. (4), we have implicitly assumed that the confinement potential is longitudinal (i.e., independent of $c$ ).

Note that the spin of the electron is disregarded in this analysis. One reason for this is that the effect of HF on the electron spin in CNT QDs has already been described in Refs. [9] and [26]. On the other hand, incorporating spin-orbit interaction and a homogeneous external magnetic field in the envelope-function Hamiltonian, (2), would modify the longitudinal envelope function $\psi(t)$ of Eq. (4) and render it spin and valley dependent (see, e.g., Appendix B.5 in Ref. [2]). We neglect these modifications, as they are expected to be small as long as the spin-orbit and Zeeman energy scales are small compared to the orbital level spacing of the QD.

The effective Hamiltonian describing the nuclear-spinelectron-valley interaction is obtained via first-order degenerate perturbation theory, i.e., by projecting $H_{\mathrm{OHF}}$ to the two-dimensional subspace spanned by $\Psi_{K}$ and $\Psi_{K^{\prime}}$ :

$$
H_{\mathrm{OHF}}^{\text {(eff) }} \equiv P H_{\mathrm{OHF}} P=\frac{1}{2} \tau_{3} \sum_{\alpha=x, y, z} C_{\alpha} I_{\alpha},
$$

where Eqs. (3) and (4) and $P \equiv\left|\Psi_{K}\right\rangle\left\langle\Psi_{K}|+| \Psi_{K^{\prime}}\right\rangle\left\langle\Psi_{K^{\prime}}\right|$ were used, $\tau_{3}$ has been redefined as $\tau_{3} \equiv\left|\Psi_{K}\right\rangle\left\langle\Psi_{K}|-| \Psi_{K^{\prime}}\right\rangle\left\langle\Psi_{K^{\prime}}\right|$, 
and

$$
C_{\alpha}\left(z_{0}\right)=\frac{e v_{\mathrm{F}}}{\pi R} \int_{-\infty}^{\infty} d t|\psi(t)|^{2} \int_{0}^{2 \pi R} d c \epsilon_{\alpha \beta \gamma} v_{\beta}\left(\boldsymbol{r}-\boldsymbol{r}_{0}\right) \hat{c}_{\gamma}
$$

Note that the last integral $\left(\int d c \ldots\right)$ is proportional to the magnetic flux that pierces the circular cross section of the CNT at height $t$ in the presence of a (classical) nuclear spin that is aligned with axis $\alpha$.

Now we evaluate the coupling strengths in Eq. (6), in the case where the length scale $\lambda$ of the spatial variation of the longitudinal envelope function $\psi(z)$ exceeds the radius $R$ of the CNT. This is the relevant case for experiments that are done with few electrons confined in an $~ 100$-nm-long QD in a CNT with radius $R \sim 1 \mathrm{~nm}$. For this case, we show that

$$
\begin{aligned}
& C_{x}\left(z_{0}\right) \approx-2 e v_{\mathrm{F}} \frac{\mu_{0} g_{N} \mu_{N}}{4 \pi}\left[\psi^{*}\left(z_{0}\right) \psi^{\prime}\left(z_{0}\right)+\text { c.c. }\right], \\
& C_{y}\left(z_{0}\right)=0, \\
& C_{z}\left(z_{0}\right) \approx 2 e v_{\mathrm{F}} \frac{\mu_{0} g_{N} \mu_{N}}{4 \pi} \frac{1}{R}\left|\psi\left(z_{0}\right)\right|^{2},
\end{aligned}
$$

where $\psi^{\prime}$ is the derivative of $\psi$ with respect to the longitudinal coordinate. Equation (5) together with Eq. (7) forms the central result of this work. The dependence of the three coupling strengths $C_{x, y, z}$ on the longitudinal position $z_{0}$ of the nuclear spin is shown in Fig. 2, for the case of a CNT with radius $R=1 \mathrm{~nm}$ and a Gaussian longitudinal envelope function

$$
\psi(t)=\frac{1}{\pi^{1 / 4} \sqrt{L}} e^{-\frac{t^{2}}{2 L^{2}}}
$$

with confinement length $L=20 \mathrm{~nm}$. In Fig. 2, lines show the analytical results of Eq. (7), whereas black circles [(red) squares] were obtained by performing the integral of Eq. (6) numerically for $\alpha=x[\alpha=z]$.

An interpretation of Eq. (7) is as follows. If the nuclear spin is aligned with the CNT axis, then it induces an energy splitting $C_{z}$ between the two valley states (i.e., a valley splitting) of the

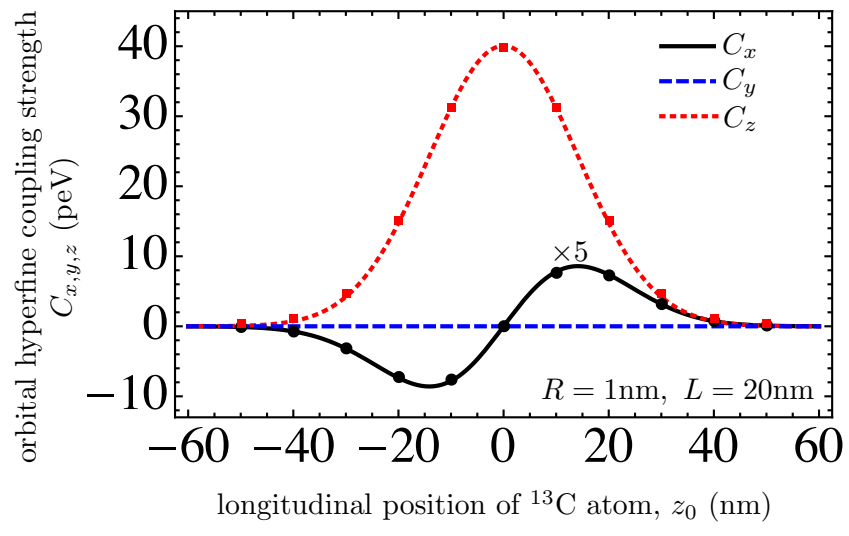

FIG. 2. (Color online) Orbital hyperfine coupling strengths as functions of the nuclear-spin position in a carbon nanotube quantum dot. The plot corresponds to a Gaussian longitudinal envelope function [Eq. (8)]. Lines show the analytical results in Eq. (7), whereas black circles [(red) squares] are obtained via numerical integration of Eq. (6) for $\alpha=x[\alpha=z]$. Note that $C_{x}$ was multiplied by 5. Axes $x$, $y$, and $z$ are defined in Fig. 1 . electron. If the nuclear spin is aligned radially, then it induces a valley splitting $C_{x}$, which is typically much smaller than in the former case, because $\left(\partial_{z} \psi\right)\left(z_{0}\right) \sim \psi\left(z_{0}\right) / \lambda$ is much smaller than $\psi\left(z_{0}\right) / R$ due to the assumed length-scale mismatch $R \ll \lambda$. Finally, if the nuclear spin is aligned orthoradially (i.e., perpendicular to the axial and radial directions), then it does not induce valley splitting. Alternatively, Eqs. (5) and (7) can be interpreted in terms of an effective magnetic field that acts on the nuclear spin and determined by the valley state of the electron. This effective magnetic field has an axial as well as a much smaller radial component, and it has no orthoradial component.

The three coupling strengths $C_{x, y, z}$ expressed in Eq. (7) have qualitatively different dependencies on the longitudinal envelope function $\psi$. A simple understanding of these differences is gained using the relation between the coupling strengths $C_{\alpha}$ and the magnetic fluxes piercing the CNT cross sections, discussed after Eq. (6). Figure 3 displays characteristic magnetic field lines piercing circular cross sections of the CNT that are positioned symmetrically with respect to the nuclear spin position, for three alignments of the nuclear spin. For a radially aligned nuclear spin [Fig. 3(a)], the fluxes piercing the two cross sections of the tube (orange) are identical in magnitude but differ in sign. Therefore, a homogeneous longitudinal envelope function $\psi(t)$ would imply a zero coupling strength $C_{x}$, since the flux contributions of the two cross sections would cancel each other in the $\int d t$ integral of Eq. (6). The inhomogeneity of $\psi(t)$, i.e., the finiteness of $\psi^{\prime}$, prevents this cancellation and allows for a finite coupling strength $C_{x}$; this is reflected by the dependence $C_{x} \propto \psi^{\prime}$ of Eq. (7a). For an axially aligned nuclear spin [Fig. 3(c)], the fluxes piercing the two circular cross sections (orange) of the CNT are identical in sign (and also in magnitude), hence the cancellation affecting $C_{x}$ is not relevant for $C_{z}$. Finally, for an orthoradially aligned nuclear spin [Fig. 3(b)], the magnetic flux piercing each of the two circular cross sections (orange) of the tube is 0, explaining Eq. (7b).

Results (5) and (7) have the following implications.

(i) In the considered range $\lambda \gg R$, the OHF-induced nuclear-spin-electron-valley interaction is essentially of Ising type, $\propto \tau_{3} I_{z}$. The correction of the form $\propto \tau_{3} I_{x}$ is small since $C_{x} \ll C_{z}$. Note that the coupling strength $C_{x}$ might gain importance in the case $\lambda \sim R$, e.g., in ultrashort CNT QDs [38-40] or in QDs where the electron occupies a highly excited, short-wavelength longitudinal mode.

(ii) Even though OHF is long-range in principle, our leading-order result, $(7 \mathrm{c})$, suggests that it is essentially local under our assumptions, since the strength of the resulting nuclear-spin-electron-valley interaction is determined by the value of the electronic envelope function at the position of the nucleus. In other words, the result, $(7 \mathrm{c})$, affirms that, for practical purposes, the envelope-function Hamiltonian $H_{\mathrm{OHF}}$ can be replaced with

$$
\tilde{H}_{\mathrm{OHF}}=e v_{\mathrm{F}} \mu_{0} g_{N} \mu_{N} \delta\left(c-c_{0}\right) \delta\left(t-t_{0}\right) \frac{\tau_{3}}{2} I_{z},
$$

since $P H_{\mathrm{OHF}} P=P \tilde{H}_{\mathrm{OHF}} P$. Therefore, an atomic coupling strength $A$ of the OHF can be defined via

$$
\tilde{H}_{\mathrm{OHF}}=\frac{\Omega_{\mathrm{cell}}}{2} A \delta\left(c-c_{0}\right) \delta\left(t-t_{0}\right) \frac{\tau_{3}}{2} I_{z},
$$


(a)

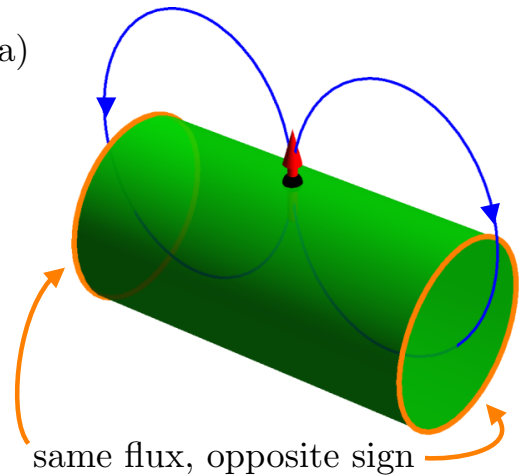

(b)

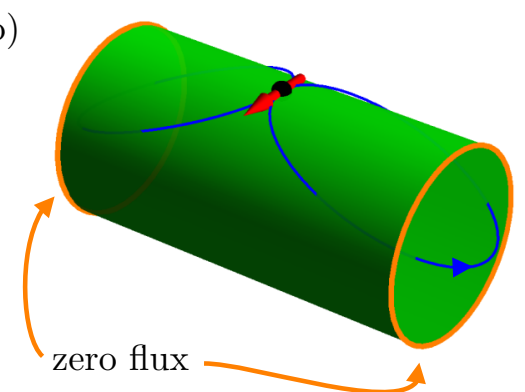

(c)

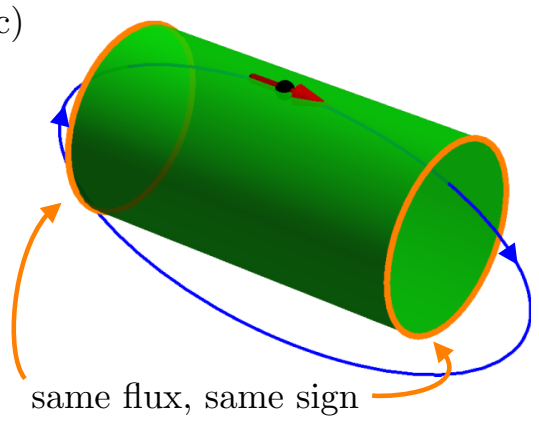

FIG. 3. (Color online) Nuclear-spin-induced magnetic field lines and magnetic fluxes. The black circle [with (red) arrow] represents the position [alignment] of the nuclear spin. The thin (blue) lines touching the nuclear spin are schematic magnetic field lines, and (blue) arrowheads on them indicate their directionality. The (orange) circles represent two symmetrically positioned circular cross sections of the tube. (a) For a radially aligned nuclear spin, the fluxes piercing the two cross sections (orange) are identical in magnitude but differ in sign. (b) For an orthoradially aligned nuclear spin, the fluxes piercing both cross sections (orange) are 0 . (c) For an axially aligned nuclear spin, the fluxes piercing the cross sections (orange) are identical in magnitude and sign.

in analogy with, e.g., the atomic coupling strength of Fermi contact HF in GaAs (see Eq. (2) of Ref. [8]). Here $\Omega_{\text {cell }} \approx$ $5.24 \AA^{2}$ is the area of the graphene unit cell. The atomic coupling strength $A$ can be deduced from Eqs. (9) and (10):

$$
A \approx \frac{2 e v_{\mathrm{F}} \mu_{0} g_{N} \mu_{N}}{\Omega_{\text {cell }}} \approx 0.34 \mu \mathrm{eV},
$$

where $v_{\mathrm{F}}=10^{6} \mathrm{~m} / \mathrm{s}$ was assumed [2].

(iii) The estimated atomic coupling strength, (11), of the OHF-induced nuclear-spin-electron-valley interaction is comparable to the atomic coupling strength of the combined Fermi contact and dipolar spin HF $[9,23]$. This result has the following consequences. (1) In order to provide an accurate assessment of any property or functionality of a CNT-based electronic spin-valley qubit $[21,22,41-43]$, the Fermi contact, dipolar, and orbital contributions should be treated on an equal footing. (2) Nuclear spins in a CNT QD will induce inhomogeneous dephasing of an electronic valley qubit on a time scale similar to the inhomogeneous dephasing time of a spin qubit or spin-valley qubit. We present a detailed analysis of the latter point in Sec. III.

For completeness we also provide the OHF Hamiltonian describing the nuclear-spin-electron-valley interaction in the presence of more than one nuclear spins $\boldsymbol{I}_{k}$. Here, $k \in 1 \ldots N$ where $N$ is the number of atoms interacting with the electron in the QD, and $\boldsymbol{I}_{k}$ is the spin-1/2 nuclear spin operator if site $k$ has a ${ }^{13} \mathrm{C}$ atom and 0 otherwise. The $k$ th atom is assumed to be located at the position specified by the circumferential $c_{k}$ and longitudinal $t_{k}$ coordinates, corresponding to the real-space position $\boldsymbol{r}_{k} \equiv\left(x_{k}, y_{k}, z_{k}\right)=\left(R \cos \left(c_{k} / R\right), R \sin \left(c_{k} / R\right), t_{k}\right)$. The effective OHF Hamiltonian, (5), then couples $\tau_{3}$ with the axial $I_{k z}$ and radial $\cos \left(c_{k} / R\right) I_{k x}+\sin \left(c_{k} / R\right) I_{k y}$ components of each nuclear spin $\boldsymbol{I}_{k}$, i.e.,

$$
\begin{aligned}
H_{\mathrm{OHF}}^{(\mathrm{eff})}= & \frac{1}{2} \tau_{3} \sum_{k=1}^{N}\left\{C_{z}\left(t_{k}\right) I_{k z}\right. \\
& \left.+C_{x}\left(t_{k}\right)\left[\cos \left(c_{k} / R\right) I_{k x}+C_{x}\left(t_{k}\right) \sin \left(c_{k} / R\right) I_{k y}\right]\right\},
\end{aligned}
$$

with $C_{x}$ and $C_{z}$ given in Eq. (7). By defining the local orbital hyperfine tensor $M_{k}$ as

$$
M_{k}=\left(\begin{array}{ccc}
0 & 0 & 0 \\
0 & 0 & 0 \\
C_{x}\left(t_{k}\right) \cos \left(c_{k} / R\right) & C_{x}\left(t_{k}\right) \sin \left(c_{k} / R\right) & C_{z}\left(t_{k}\right)
\end{array}\right),
$$

the effective OHF Hamiltonian can be compactly written as

$$
H_{\mathrm{OHF}}^{(\mathrm{eff})}=\frac{1}{2} \boldsymbol{\tau} \sum_{k=1}^{N} M_{k} \boldsymbol{I}_{k} .
$$

This section is concluded by proving Eq. (7). First, we prove Eq. (7a) by evaluating $C_{x}$ using Eq. (6), the definition of $v$ via Eq. (1), and the definition of $\hat{\boldsymbol{c}}$ given following Eq. (2). After introducing the dimensionless quantities $\varphi=c / R$ and $\zeta=\frac{t-z_{0}}{R}$, we find

$$
\begin{aligned}
C_{x}\left(z_{0}\right)= & -\frac{e v_{\mathrm{F}}}{\pi R} \frac{\mu_{0} g_{N} \mu_{N}}{4 \pi} \int_{-\infty}^{\infty} d \zeta\left|\psi\left(z_{0}+R \zeta\right)\right|^{2} \\
& \times \int_{0}^{2 \pi} d \varphi \frac{\zeta \cos (\varphi)}{\left(2-2 \cos (\varphi)+\zeta^{2}\right)^{3 / 2}} .
\end{aligned}
$$

Note that although the integrand is singular at the position of the nuclear spin, i.e., for $(c, t)=\left(0, z_{0}\right)$, that is, for $(\varphi, \zeta)=$ $(0,0)$, the integral does converge, similarly to the case of OHF in graphene [35]. Importantly, the integrand in the second line of Eq. (15) decays for $\zeta \gg 1$ as $\sim 1 / \zeta^{2}$, suggesting that the integral is dominated by the range $|\zeta| \lesssim 1$, that is, by the spatial range $\left|z-z_{0}\right| \lesssim R$. Since this spatial range is narrow in comparison with the length scale $\lambda$ characterizing the spatial variation of the longitudinal envelope function $\psi$, 
we can estimate the value of the integral by expanding the term $|\psi|^{2}$ up to first order in $\zeta:\left|\psi\left(z_{0}+R \zeta\right)\right|^{2} \approx\left|\psi\left(z_{0}\right)\right|^{2}+$ $R \zeta\left[\psi^{*}\left(z_{0}\right) \psi^{\prime}\left(z_{0}\right)+\right.$ c.c.]. The integral containing the zerothorder term vanishes as its integrand is an odd function of $\zeta$. The integral containing the first-order term is finite, though, providing the estimate, Eq. (7a). Technically, the $C_{y}=0$ result in Eq. (7b) follows from the fact that the $\alpha=y$ integrand in Eq. (6) is an antisymmetric function of $c \in[0,2 \pi R]$ with respect to $c=\pi R$. Finally, the derivation of Eq. (7c) is similar to that of Eq. (7a). The difference here is that the integral will be dominated by the zeroth-order term in the $\zeta$ expansion of $\left|\psi\left(z_{0}+R \zeta\right)\right|^{2}$, hence higher order terms can be neglected.

\section{INHOMOGENEOUS DEPHASING OF A VALLEY QUBIT}

HF leads to information loss via decoherence for spin qubits in conventional semiconductors $[8,44]$ as well as in carbon nanostructures $[9,29,30]$. One form of decoherence is inhomogeneous dephasing, which arises due to a nuclear-spininduced random component in the qubit's Larmor frequency and is characterized by the time scale $T_{2}^{*}$, usually called the inhomogeneous dephasing time. To our knowledge, the $T_{2}^{*}$ of valley qubits [45-49] due to HF has not yet been investigated. Based on the result in the previous section, here we evaluate $T_{2}^{*}$ for a valley qubit formed in the conduction-band ground state of a CNT QD, as a function of the CNT radius $R$, the QD length $L$, and the abundance $v \in[0,1]$ of ${ }^{13} \mathrm{C}$ atoms.

We assume that the valley states of the single electron forming the valley qubit are energy-split $\left(\hbar \omega_{L}\right)$ by a longitudinal magnetic field and/or spin-orbit interaction, and the qubit is tuned far away from the $K-K^{\prime}$ anticrossing [50,51] caused by valley mixing. The valley qubit is prepared in a superposition state, $\Psi(t=0)=\frac{1}{\sqrt{2}}\left(\left|\Psi_{K}\right\rangle+\left|\Psi_{K^{\prime}}\right\rangle\right)$. It interacts with the nuclear spin bath, which is completely disordered to a good approximation. The Hamiltonian reads

$$
H=\frac{1}{2} \hbar \omega_{L} \tau_{3}+H_{\mathrm{OHF}}^{(\mathrm{eff})}
$$

where $H_{\mathrm{OHF}}^{\text {(eff) }}$ is given in Eq. (14), and we neglect $C_{x}$.

Following Merkulov et al. [8], we disregard the slow dynamics of the nuclear spin bath and describe the nuclear spins as being frozen during the time evolution of the electronic valley state. The influence of the nuclear spin ensemble is expressed via the HF-induced random correction $\delta \omega=$ $\frac{1}{\hbar} \sum_{k, \alpha} M_{k ; 3, \alpha} I_{k, \alpha} \approx \frac{1}{\hbar} \sum_{k} C_{z}\left(z_{k}\right) I_{k, z}$ of the valley Larmor frequency $\omega_{L}$. In the presence of many nuclear spins, the correction $\delta \omega$ can be regarded as a Gaussian random variable with the following mean and variance:

$$
\begin{gathered}
\overline{\delta \omega} \equiv \frac{1}{\hbar} \overline{\sum_{k} C_{z}\left(z_{k}\right) I_{k, z}}=0, \\
\sigma^{2} \equiv \overline{(\delta \omega)^{2}}=\frac{v}{4} \frac{1}{\hbar} \sum_{k} C_{k}^{2}\left(z_{k}\right) .
\end{gathered}
$$

Here the overline refers to both ensemble averaging for the nuclear spin states and disorder averaging for the possible spatial configurations of the spin-carrying nuclei. Correspondingly, we used $\overline{I_{k, \alpha}}=0$ and $\overline{I_{k, \alpha} I_{k^{\prime}, \alpha^{\prime}}}=\frac{v}{4} \delta_{k k^{\prime}} \delta_{\alpha \alpha^{\prime}}$, and $v$ is the abundance of spin-carrying nuclei.
The polarization vector of the valley qubit in the initial state $\Psi(0)$ is $\boldsymbol{p}(t=0) \equiv\langle\Psi(0)|\boldsymbol{\tau}| \Psi(0)\rangle=(1,0,0)^{T}$. A straightforward calculation [8] shows that the time evolution of the valley polarization $\boldsymbol{p}(t)$, averaged for the random nuclear-spin configurations, reads

$$
\overline{\boldsymbol{p}(t)} \equiv \overline{\langle\Psi(t)|\boldsymbol{\tau}| \Psi(t)\rangle}=\left(\begin{array}{c}
\cos \left(\omega_{L} t\right) \\
\sin \left(\omega_{L} t\right) \\
0
\end{array}\right) e^{-\left(t / T_{2}^{*}\right)^{2}},
$$

where

$$
T_{2}^{*}=\sqrt{2} / \sigma=\frac{1}{\sqrt{v}} \frac{2 \sqrt{2} \hbar}{\sqrt{\sum_{k} C_{z}^{2}\left(z_{k}\right)}} .
$$

For a box-type longitudinal envelope function, i.e., if $\psi(z)=$ $1 / \sqrt{L}$ within a QD of length $L$, we find

$$
T_{2}^{*} \approx \frac{1}{\sqrt{v}} 2^{3 / 2} \sqrt{\pi} \frac{\sqrt{\Omega_{\mathrm{cell}}} \hbar}{e v_{\mathrm{F}} \mu_{0} g_{N} \mu_{N}} \sqrt{L R},
$$

where Eqs. (7c) and (20) were used. For a natural (nonisotope-enriched) CNT QD containing $N=6 \times 10^{5}$ atoms, we estimate $T_{2}^{*} \approx 266 \mu \mathrm{s}$, comparable to the theoretically estimated spin dephasing time [9]. This is not surprising, considering that the orbital HF atomic coupling strength estimated in the previous section was also comparable to the spin HF (combined Fermi contact and dipolar) atomic coupling strength.

For the Gaussian longitudinal envelope function defined in Eq. (8), which provides a more realistic description of the ground-state orbital of a QD with parabolic electrostatic confinement, we find the same parameter dependence as for the box-model wave function, with slightly different prefactors:

$$
T_{2}^{*} \approx \frac{1}{\sqrt{v}} 2^{7 / 4} \pi^{3 / 4} \frac{\sqrt{\Omega_{\text {cell }}} \hbar}{e v_{\mathrm{F}} \mu_{0} g_{N} \mu_{N}} \sqrt{L R} .
$$

Note that if the relatively low coupling strength $C_{x}$ corresponding to a radially aligned nuclear spin is taken into account, then the inhomogeneous dephasing time is $\left(1-\frac{R^{2}}{2 L^{2}}\right)$ times the right-hand side of Eq. (22), i.e., the correction due to $C_{x}$ is second order in the small quantity $R / L$. The coupling strength $C_{x}$ might gain importance and significantly contribute to the $T_{2}^{*}$ in ultrashort CNT QDs [38-40], where $L \sim R$, or for electrons that occupy a highly excited longitudinal mode of a QD.

The interpretation of the results, (21) and (22), is straightforward. An increasing ${ }^{13} \mathrm{C}$ abundance $v$ leads to a shorter $T_{2}^{*}$, and the inverse-square-root dependence on $v$ originates from the completely randomized character of the nuclear spin bath. The inverse linear dependence of $T_{2}^{*}$ on the parameters setting the interaction strength $\left(e, v_{F}, \mu_{0}, g_{N}, \mu_{N}\right)$ and the square-root dependence on the geometrical parameters $R$ and $L$ are natural consequences of the parametric dependencies of the orbital HF coupling strengths in Eq. (7).

\section{DISCUSSION}

(i) A natural consequence of our results is that the nuclearspin-electron-valley interaction arising from OHF contributes to the dephasing of spin-valley qubits in CNTs [22,41]. To our knowledge, this contribution has not been analyzed to date. As 
the strength of this interaction is comparable to those of the nuclear-spin-electron-spin [9,23] and nuclear-spin-electronvalley [26] interactions arising from the Fermi contact and dipolar mechanisms, it is expected that the hyperfine-limited inhomogeneous dephasing time of a spin-valley qubit has a scale similar to that of the spin qubit (see Ref. [9]) and the valley qubit (see Sec. III). A detailed calculation of $T_{2}^{*}$ of the spin-valley qubit, which could quantify, e.g., the dependence of $T_{2}^{*}$ on the direction of the homogeneous magnetic field, has yet to be done.

(ii) We emphasize that valley-qubit coherence might be affected by mechanisms other than OHF. For example, spin-independent potential disorder, similarly to the case of silicon-based heterostructure QDs [52-54], makes the valley qubit susceptible to electric fields [46], including electrical fluctuations caused by phonons or nearby electrodes. In addition, if a CNT valley qubit is tuned by an axial magnetic field to the $K-K^{\prime}$ anticrossing (e.g., to $0.11 \mathrm{~T}$ in Fig. 2(e) of Ref. [51]), then valley mixing due to Fermi contact and dipolar HF [26] can also induce qubit dephasing. Exploring the competition and interplay of various valley-qubit decoherence mechanisms is an interesting future direction.

(iii) Importantly, our present effort, which completes the theoretical description of the basic hyperfine mechanisms for CNT QDs, does not explain the comparatively strong hyperfine coupling strength deduced from the experiments in Refs. [16] and [17]. Further discussion of the relation of these experiments and the theory of HF can be found in Secs. IV and V of Ref. [2].

(iv) HF between electronic spin qubits and nuclear spins can be harmful, as described above, from the quantum information perspective. It can also be an asset though: in principle, nuclear spins can be used as long-lived quantum memories [11-14], and information transfer between the electronic and the nuclear degrees of freedom can be mediated by HF. Furthermore, enrichment (purification) of the ${ }^{13} \mathrm{C}$ abundance is a feasible way [15-17] to enhance (suppress) hyperfine effects.

(v) In certain inversion-symmetry-broken two-dimensional multivalley materials, such as monolayer transition-metal dichalcogenides [55] or gapped graphene [56], the electronic states acquire a finite valley-dependent magnetic moment. This magnetic moment is inherently coupled with the nuclear spins of the crystal lattice via OHF; therefore, if an electron is confined in a QD in these materials [57,58], then its operation as a valley qubit or as a spin-valley qubit will be influenced by the OHF-induced nuclear-spin-electron-valley interaction in a similar fashion as in a CNT. The OHF and its consequences in such two-dimensional materials have yet to be explored.

In conclusion, we have shown that $\mathrm{OHF}$ couples the nuclear spins residing in a CNT QD and the valley degree of freedom of the electron confined in the QD. We have provided a quantitative analysis of this interaction and found that it is essentially a local Ising-type interaction, which is as strong as the nuclearspin-electron-spin HFs (Fermi contact and dipolar). As an application, we evaluated the hyperfine-limited inhomogeneous dephasing time of a single-electron valley qubit, which was found to be in the $\sim 100-\mu$ s range, similar to theoretical estimates for CNT-based spin qubits but much longer than the measured $T_{2}^{*}$ of single-electron spin-valley qubits.

\section{ACKNOWLEDGMENTS}

We thank P. Boross, G. Burkard, B. Dóra, P. Nagy, M. Rudner, F. Simon, Á. Szabados, and G. Tichy for useful discussions. We acknowledge funding from EU Marie Curie Career Integration Grant No. CIG-293834 (CarbonQubits), OTKA Grant No. PD 100373, and EU ERC Starting Grant No. CooPairEnt 258789. A.P. was supported by the János Bolyai Scholarship of the Hungarian Academy of Sciences.
[1] F. Kuemmeth, H. Churchill, P. Herring, and C. Marcus, Mater. Today 13, 18 (2010).

[2] E. Laird, F. Kuemmeth, G. Steele, K. Grove-Rasmussen, J. Nygard, K. Flensberg, and L. P. Kouwenhoven, arXiv:1403.6113.

[3] D. Loss and D. P. DiVincenzo, Phys. Rev. A 57, 120 (1998).

[4] R. Hanson, L. P. Kouwenhoven, J. R. Petta, S. Tarucha, and L. M. K. Vandersypen, Rev. Mod. Phys. 79, 1217 (2007).

[5] R. Hanson and D. D. Awschalom, Nature 453, 1043 (2008).

[6] D. D. Awschalom, L. C. Bassett, A. S. Dzurak, E. L. Hu, and J. R. Petta, Science 339, 1174 (2013).

[7] F. H. L. Koppens, C. Buizert, K. J. Tielrooij, I. T. Vink, K. C. Nowack, T. Meunier, L. P. Kouwenhoven, and L. M. K. Vandersypen, Nature 442, 766 (2006).

[8] I. A. Merkulov, A. L. Efros, and M. Rosen, Phys. Rev. B 65, 205309 (2002).

[9] J. Fischer, B. Trauzettel, and D. Loss, Phys. Rev. B 80, 155401 (2009).

[10] B. E. Kane, Nature 393, 133 (1998).

[11] J. M. Taylor, C. M. Marcus, and M. D. Lukin, Phys. Rev. Lett. 90, 206803 (2003).
[12] M. V. G. Dutt, L. Childress, L. Jiang, E. Togan, J. Maze, F. Jelezko, A. S. Zibrov, P. R. Hemmer, and M. D. Lukin, Science 316, 1312 (2007).

[13] G. D. Fuchs, G. Burkard, P. V. Klimov, and D. D. Awschalom, Nature Phys. 7, 789 (2011).

[14] J. J. Pla, K. Y. Tan, J. P. Dehollain, W. H. Lim, J. J. L. Morton, F. A. Zwanenburg, D. N. Jamieson, A. S. Dzurak, and A. Morello, Nature 496, 334 (2013).

[15] F. Simon, C. Kramberger, R. Pfeiffer, H. Kuzmany, V. Zólyomi, J. Kürti, P. M. Singer, and H. Alloul, Phys. Rev. Lett. 95, 017401 (2005).

[16] H. O. H. Churchill, F. Kuemmeth, J. Harlow, A. J. Bestwick, E. I. Rashba, K. Flensberg, C. H. Stwertka, T. Taychatanapat, S. K. Watson, and C. M. Marcus, Phys. Rev. Lett. 102, 166802 (2009).

[17] H. O. H. Churchill, A. J. Bestwick, J. W. Harlow, F. Kuemmeth, D. Marcos, C. H. Stwertka, S. K. Watson, and C. M. Marcus, Nature Phys. 5, 321 (2009).

[18] B. Braunecker, P. Simon, and D. Loss, Phys. Rev. Lett. 102, 116403 (2009).

[19] B. Braunecker, P. Simon, and D. Loss, Phys. Rev. B 80, 165119 (2009). 
[20] C. P. Scheller, T.-M. Liu, G. Barak, A. Yacoby, L. N. Pfeiffer, K. W. West, and D. M. Zumbühl, Phys. Rev. Lett. 112, 066801 (2014).

[21] F. Pei, E. A. Laird, G. A. Steele, and L. P. Kouwenhoven, Nat. Nanotech. 7, 630 (2012).

[22] E. A. Laird, F. Pei, and L. P. Kouwenhoven, Nat. Nanotech. 8, 565 (2013).

[23] O. V. Yazyev, Nano Lett. 8, 1011 (2008).

[24] C. H. Pennington and V. A. Stenger, Rev. Mod. Phys. 68, 855 (1996).

[25] A. Kiss, A. Pályi, Y. Ihara, P. Wzietek, H. Alloul, P. Simon, V. Zólyomi, J. Koltai, J. Kürti, B. Dóra et al., Phys. Rev. Lett. 107, 187204 (2011).

[26] A. Pályi and G. Burkard, Phys. Rev. B 80, 201404 (2009).

[27] A. A. Reynoso and K. Flensberg, Phys. Rev. B 84, 205449 (2011).

[28] A. A. Reynoso and K. Flensberg, Phys. Rev. B 85, 195441 (2012).

[29] M. Fuchs, V. Rychkov, and B. Trauzettel, Phys. Rev. B 86, 085301 (2012).

[30] M. Fuchs, J. Schliemann, and B. Trauzettel, Phys. Rev. B 88, 245441 (2013).

[31] A. Abragam, The Principles of Nuclear Magnetism (Oxford University Press, New York, 1961).

[32] Y. Yafet, J. Phys. Chem. Solids 21, 99 (1961).

[33] S. Latil, L. Henrard, C. Goze Bac, P. Bernier, and A. Rubio, Phys. Rev. Lett. 86, 3160 (2001).

[34] C. Goze-Bac, S. Latil, P. Lauginie, V. Jourdain, J. Conard, L. Duclaux, A. Rubio, and P. Berniera, Carbon 40, 1825 (2002).

[35] B. Dóra and F. Simon, Phys. Rev. Lett. 102, 197602 (2009).

[36] H. Ajiki and T. Ando, J. Phys. Soc. Jpn. 62, 1255 (1993).

[37] E. D. Minot, Y. Yaish, V. Sazonova, and P. L. McEuen, Nature 428, 536 (2004).

[38] X. Sun, S. Zaric, D. Daranciang, K. Welsher, Y. Lu, X. Li, and H. Dai, J. Am. Chem. Soc. 130, 6551 (2008).
[39] J. O. Island, V. Tayari, S. Yigen, A. C. McRae, and A. R. Champagne, Appl. Phys. Lett. 99, 243106 (2011).

[40] P. Petit, C. Feuillet-Palma, M. L. Della Rocca, and P. Lafarge, Phys. Rev. B 89, 115432 (2014).

[41] K. Flensberg and C. M. Marcus, Phys. Rev. B 81, 195418 (2010).

[42] G. Széchenyi and A. Pályi, Phys. Rev. B 89, 115409 (2014).

[43] E. N. Osika, A. Mreńca, and B. Szafran, Phys. Rev. B 90, 125302 (2014).

[44] W. A. Coish and J. Baugh, Phys. Status Solidi B 246, 2203 (2009).

[45] P. Recher, B. Trauzettel, A. Rycerz, Y. M. Blanter, C. W. J. Beenakker, and A. F. Morpurgo, Phys. Rev. B 76, 235404 (2007).

[46] A. Pályi and G. Burkard, Phys. Rev. Lett. 106, 086801 (2011).

[47] G. Y. Wu, N.-Y. Lue, and L. Chang, Phys. Rev. B 84, 195463 (2011).

[48] D. Culcer, A. L. Saraiva, B. Koiller, X. Hu, and S. D. Sarma, Phys. Rev. Lett. 108, 126804 (2012).

[49] N. Rohling and G. Burkard, New J. Phys. 14, 083008 (2012).

[50] D. V. Bulaev, B. Trauzettel, and D. Loss, Phys. Rev. B 77, 235301 (2008).

[51] F. Kuemmeth, S. Ilani, D. C. Ralph, and P. L. McEuen, Nature 452, 448 (2008).

[52] M. Friesen and S. N. Coppersmith, Phys. Rev. B 81, 115324 (2010).

[53] D. Culcer, X. Hu, and S. Das Sarma, Phys. Rev. B 82, 205315 (2010).

[54] J. K. Gamble, M. A. Eriksson, S. N. Coppersmith, and M. Friesen, Phys. Rev. B 88, 035310 (2013).

[55] D. Xiao, G.-B. Liu, W. Feng, X. Xu, and W. Yao, Phys. Rev. Lett. 108, 196802 (2012).

[56] D. Xiao, W. Yao, and Q. Niu, Phys. Rev. Lett. 99, 236809 (2007).

[57] A. Kormányos, V. Zólyomi, N. D. Drummond, and G. Burkard, Phys. Rev. X 4, 011034 (2014).

[58] P. Recher, J. Nilsson, G. Burkard, and B. Trauzettel, Phys. Rev. B 79, 085407 (2009). 